\title{
Incidence, Socio-demographic Characteristics and Predisposing Factors of Ectopic Pregnancy attended at a Private Tertiary Care Hospital in Dhaka City
}

\author{
Naheed Fatema', Sherajum Munira ${ }^{2}$, KAM Mahbub Hasan³, Zannatul Ferdosh', \\ Nasim Iftekhar Mahmud ${ }^{5}$, Shahla Khatun ${ }^{6}$
}

\begin{abstract}
${ }^{1}$ Resident Surgeon, Department of Obstetrics and Gynecology, Bangladesh Medical College Hospital, Dhaka, Bangladesh; ${ }^{2}$ Assistant Registrar,Department of Obstetrics and Gynecology, Kurmitola General Hospital, Dhaka, Bangladesh; ${ }^{3}$ Medical Officer, Department of Cardiology, National Institute of Cardiovascular Diseases, Dhaka, Bangladesh; ${ }^{4}$ Medical Officer, Lakshmipur District Hospital, Dhaka, Bangladesh; ${ }^{5}$ Junior Consultant (Anesthesiology), Lakshmipur District Hospital,Lakshmipur, Bangladesh; ${ }^{6}$ National Professor \& Chairman, Green Life Medical College Hospital, Dhaka, Bangladesh
\end{abstract}

[Received: 12 August 2019; Accepted: 20 October 2019; Published: 1 January 2020]

\begin{abstract}
Background: Ectopic pregnancy is frequently occurs among pregnant women. Objective: The purpose of the present study was to see the incidence and socio-demographic characteristics of ectopic pregnancy patients. Methodology: This cross-sectional study was carried out in the inpatient Department of Obstetrics and Gynaecology at Bangladesh Medical College Hospital, Dhaka, Bangladesh from January 2009 to December 2009 for a period of one (1) year. All clinically suspected cases of ectopic pregnancy within the reproductive age admitted in the department of Obstetrics and Gynaecology at Bangladesh Medical College Hospital, Dhaka were included in the present study. After taking detailed history with particular scrutinization of the menstrual, obstetric and contraceptive history, a thorough physical examination was performed. The ectopic pregnancy was confirmed by laboratory and imaging technique. Result: The incidence of ectopic pregnancy in $\mathrm{BMCH}$ was $2.16 \%$ cases. The mean age with SD of the study population was $24.0 \pm 2.87$ years. The ectopic pregnancy was closely related with high parity and it had been found that para- 2 was the most common which was $20(44 \%)$ cases.Pelvic infection $(33.0 \%)$, past history of abortion or MR $(28.0 \%)$, and history of infertility $(25.0 \%)$ were constituted the main bulk of risk factors. Only $3.0 \%$ had history of tuberculosis. Conclusion: In conclusion young para 2 women with pelvic infection or past history of abortion or MR are most commonly suffering from ectopic pregnancy. [Journal of National Institute of Neurosciences Bangladesh, 2019;6(1): 59-63]
\end{abstract}

Keywords: Incidence; socio-demographic characteristics; predisposing factors; ectopic pregnancy

Correspondence: Dr. NaheedFatema, Resident Surgeon, Departmentof Obstetrics and Gynecology, Bangladesh Medical College Hospital,Dhanmondi, Dhaka, Bangladesh;dr.naheedkoly@gmail.com; Cell no.: +8801715301006

Conflict of interest: There is no financial conflict of interest relevant to this paper to disclose.

Funding agency: This research project was not funded by any group or any institution.

Contribution to authors: Fatema N, Munira S, Hasan KAMM have contributed from the protocol preparation, data collection up to report writing. Manuscript writing was performed by Fatema N.Ferdosh Z, Mahmud NI, Khatun Shave revised the manuscript.

How to cite this article: Fatema N, Munira S,Hasan KAMM, Ferdosh Z, Mahmud NI, Khatun S.Incidence, Socio-demographic Characteristics and Predisposing Factors of Ectopic Pregnancy attended at a Private Tertiary Care Hospital in Dhaka City. J NatlInstNeurosci Bangladesh, 2020;6(1): 59-63

Copyright: (C2020. Fatema et al. Published by Journal of National Institute of Neurosciences Bangladesh. This article is published under the Creative Commons CC BY-NC License (https://creativecommons.org/licenses/by-nc/4.0/). This license permits use, distribution and reproduction in any medium, provided the original work is properly cited, and is not used for commercial purposes.

\section{Introduction}

Ectopic pregnancy presents a major health problem for women of childbearing age ${ }^{1}$. Without timely diagnosis and treatment, ectopic pregnancy can become a life-threatening situation. Ectopic pregnancy is a significant cause of maternal morbidity and mortality as well as fetal $\operatorname{loss}^{2}$. It is the leading cause of pregnancy-related death in the first trimester, and accounts for $9.0 \%$ of all pregnancy-related deaths ${ }^{3}$. In addition to the immediate morbidity caused by ectopic pregnancy, the woman's future ability of reproduction may be adverselyaffected as well.

One report demonstrated only 5 patients out of 30 survived after the abdominal operation ${ }^{4}$. Interestingly, the survival rate in patients who are left untreated is 1 
of 3 in the beginning of the $20^{\text {th }}$ century, great improvements in anesthesia, antibiotics, and blood transfusion is contributed to the decrease in the maternal mortality rate ${ }^{5}$. In the early half of the $20^{\text {th }}$ century, 200 to 400 deaths per 10,000 cases are attributed to ectopic pregnancy ${ }^{6}$. Centers for Disease Control and Prevention (CDC) has begun to record the statistics regarding ectopic pregnancy. The number of ectopic pregnancies had increased to 108,800 cases $^{7}$. Concurrently, however, the case fatality rate decreased from 35.5 deaths per 10,000 cases in 1970 to 2.6 per 10,000 cases $^{3}$.

Ectopic pregnancy is seen in about $2.0 \%$ of all pregnancies in USA, 3.0 to $4.0 \%$ worldwide incidence ${ }^{4}$. In some studies the incidence reported is as high as 16 ectopic pregnancy for 1000 cases $^{8}$. The incidence of ectopic pregnancy has risen in the past 20 years due to various reasons like predisposing factors and also better diagnostic techniques. The possible causes of increase in incidence are increased rate of PID, increased use of different contraception and assisted reproductive technology, increased rate of tubal surgical procedures ${ }^{4}$.

In another study is has been reported that the commonest cause is acute salpingitis in 50 percent of cases $^{9}$. Salpingitis causes peritubal adhesions, partial tubal lumen occlusion, intratubal adhesions, diverticula and disturbed tubal functions. Salpingitismay be due to Chlamydia, gonococcal infection, tuberculosis, post abortal, puerperal or secondary to pelvic peritonitis infection source could be extragenital also like appendicitis ${ }^{10}$. Different modes of Contraception have been blamed as they effectively reducethe uterine implantation but have no effects on tubal or ovarian implantation. Previous operation on the tubes, tubal developmental factors including mucosal defects are all responsible even chromosomal abnormalities of the conceptus can cause ectopic pregnancies. This present study was undertaken to see the incidence and socio-demographic characteristics of ectopic pregnancy patients.

\section{Methodology}

This was a descriptive type of cross-sectional study. This study was carried out in the inpatient Department of Obstetrics and Gynaecology at Bangladesh Medical College Hospital, Dhaka, Bangladesh. This study was conducted from January 2009 to December 2009 for a period of one (1) year. All clinically suspected cases of ectopic pregnancy within the reproductive age admitted in the Department of Obstetrics and Gynaecology at the Bangladesh Medical College Hospital, Dhaka, Bangladesh during the study period were included in the present study. The clinically suspected cases of ectopic pregnancy with the age group of 15 to 45 years were included in this study. Patients other than ectopic pregnancy were excluded from this study. The sampling technique was consecutive sampling technique and this purposive sampling technique was used as per inclusions and exclusion criteria. After taking detailed history with particular scrutinization of the menstrual, obstetric and contraceptive history, a thorough physical examination was performed, and ectopic pregnancy was diagnosed clinically in a large number of cases. In some cases, investigations like pregnancy test, beta-hCG and ultrasonography were done to support the clinical diagnosis. Data was collected on initial presentation, chief complaints, socio-demographic characteristics, past obstetrics and gynaecological history, history of previous surgeries (tubal, ovarian and/or uterine), history of infertility and use of ovulation induction and history of contraception was obtained. It was a pretested semi-structured questionnaire and a checklist developed by using selectedvariables according to the objectives. Laboratory findings and treatment history was recorded. The questionnaire was prepared in English. Data collection was conducted by researcher herself. All data were compiled and edited meticulously by thorough checking and rechecking. All omissions and inconsistencies were corrected and were removed methodically. All data were recorded systematically in preformed data collection form (questionnaire) and quantitative data was expressed as mean and standard deviation and qualitative data was expressed as frequency distribution and percentage. Statistical analysis was performed by using SPSS for windows version 12.0. A 95\% confidence limit was taken. Probability value $<0.05$ was considered as level of significance. Prior to the commencement of this study, the research protocol was approved by the ethical committee (Local Ethical committee) of the BangladeshMedical College Hospital, Dhaka. Informed consent was taken from the respondents prior to interview.

\section{Results}

A total number of 2138 patients were admitted at the inpatients department of Obstetrics and Gynaecology at Bangladesh Medical College Hospital, Dhaka for a 
period of one year of which 48 cases were confirmed as ectopic pregnancy. These 48 ectopic pregnancy cases were further evaluated to observe the risks factors, clinical presentations and managements with their improvements. The incidence of ectopic pregnancy in this studywas $2.16 \%$ cases (Table 1 ).

Table 1: Incidence of Ectopic Pregnancy

\begin{tabular}{lcc}
\hline Type of Patients & Frequency & Percent \\
\hline Obstetric Patients & 1833 & 86.0 \\
Abortion & 257 & 12.02 \\
Ectopic Pregnancy & 48 & 2.16 \\
Total & $\mathbf{2 1 3 8}$ & $\mathbf{1 0 0}$ \\
\hline
\end{tabular}

Majority of the cases was in 21 to 25 years age group which was $20(41.66 \%)$ cases followed by less than 20 years and 26 to 30 Years of age group which were $14(30.55 \%)$ cases and $10(19.44 \%)$ cases respectively. Only $4(8.33 \%)$ cases were in the more than 30 years age group. The mean age with SD of the study population was $24.0 \pm 2.87$ years (Table 2 ).

Table 2: Distribution of Ectopic Pregnancy Cases according to age

\begin{tabular}{lcc}
\hline Age Group & Frequency & Percent \\
\hline Less than 20 Years & 14 & 30.55 \\
21 to 25 Years & 20 & 41.66 \\
26 to 30 Years & 10 & 19.44 \\
More than 30 Years & 4 & 8.33 \\
Total & $\mathbf{4 8}$ & $\mathbf{1 0 0 . 0}$ \\
\hline
\end{tabular}

The ectopic pregnancy was closely related with high parity and it had been found that para- 2 was the most common which was $20(44 \%)$ cases followed by para 3 and para 1 which was $12(22.6 \%)$ cases and $9(16.4 \%)$ cases respectively. However, para 4 and para 0 were in $6(11.1 \%)$ cases and $4(5.5 \%)$ cases respectively (Table 3 ).

Table 3: Ectopic Pregnancy Cases According To Parity

\begin{tabular}{lcc}
\hline Number of Parity & Frequency & Percent \\
\hline 0 & 4 & 5.5 \\
1 & 9 & 16.4 \\
2 & 20 & 44.4 \\
3 & 12 & 22.6 \\
4 & 6 & 11.1 \\
Total & $\mathbf{4 8}$ & $\mathbf{1 0 0 . 0}$ \\
\hline
\end{tabular}

The distribution of cases according to predisposing factors were recorded. Pelvic infection (33.0\%), past history of abortion or MR (28.0\%), and history of infertility $(25.0 \%)$ were constituted the main bulk of risk factors. Only $3.0 \%$ had history of tuberculosis. It had been found that $6.0 \%$ patients had history of appendicitis and another $6.0 \%$ patients had history of lower abdominal surgery and $3.0 \%$ patients were IUCD user (Table 4).

Table 2: Distribution of Ectopic Pregnancy Cases according to age

\begin{tabular}{lcc}
\hline Risk Factors & Frequency & Percent \\
\hline H/O Previous abortion/MR & 12 & 27.8 \\
H/O Ectopic Pregnancy & 0 & 0.0 \\
H/O of Infertility & 8 & 25.0 \\
Pelvic Infection & 16 & 33.3 \\
Tuberculosis & 2 & 2.8 \\
Appendicitis & 4 & 5.5 \\
H/O Lower Abdominal Surgery & 4 & 5.5 \\
IUCD & 2 & 2.7 \\
Total & $\mathbf{4 8}$ & $\mathbf{1 0 0 . 0}$ \\
\hline
\end{tabular}

\section{Discussion}

Ectopic pregnancy has considerable medical importance as it is associated with maternal mortality, infertility and adverse outcome in subsequent pregnancy ${ }^{11}$. The incidence of ectopic pregnancy varies from place to place, even in the same country. Its incidence is increased in many developed countries in recent years and mortality has decreased due to significant improvement in diagnostic technology ${ }^{6}$. My study was conducted on admitted patient of $\mathrm{BMCH}$ who were diagnosed clinically as ectopic pregnancy. The incidence has been found to be 21.6 per 1000 total pregnancies during the observed period. There are different studies showing the fact that, the incidence of ectopic pregnancy in United States is increasing from 4.5 per 1000 registered pregnancy in 1970 to 19.7 per 1000 in 19922. The high figure in the developed countries has been attributed to an increased incidence of salpingitis as well as to improved diagnostic techniques ${ }^{6}$. In this study, unsafe abortion leading to pelvic inflammatory disease is the main risk factor for ectopic pregnancy. There are reports with the opinion that, high incidence of illegal abortion increases the risk of ectopic pregnancy many fold. Presumably, this increase is secondary to post-operative infection and improper performed procedures.

Ectopic pregnancy is a critical episodein a woman's life. The incidence varies from place to place, even in the same country. It is the leading cause of pregnancy-related death in the first trimester, and 
accounts for $9.0 \%$ of all pregnancy-related deaths ${ }^{2}$. Although there has been a massive increase in the incidence of ectopic pregnancy, the mortality of the disease has been static $^{8}$. The incidence of ectopic pregnancy is rising. This rise is both real as well as apparent. The real rise seems to be due to increased incidence of PID, sexually transmitted disease (STD), contraception method like IUCD and progesterone only pills and modem methods of infertility treatment like assisted reproductive technique ${ }^{12}$. The apparent rise could be because of better diagnostic facilities suchasbeta-hCG estimation and high-resolution ultrasonography. In the multicentric case control study of ectopic pregnancy in India, the incidence of ectopic pregnancies was 3.12 per 1,000 pregnancies or 3.86 per 1000 live birth ${ }^{9}$. A study from Sweden supports an association between ectopic pregnancy and preceding infection by Chlamydia. It has also been found that the reduction in the rate of chlamydial infection due to screening and treatment leads to concomitant decline in the incidence of ectopic pregnancy ${ }^{10}$. The increased uses of assisted reproductive technology have contributed to the high rate of multiple and ectopic pregnancy during the past decade ${ }^{11}$.

Generally, ectopic pregnancy may occur at any age during the reproductive period. In my study majority of patient $(42.0 \%)$ were in 21 to 25 years age group, $8.0 \%$ patients were above 30 years group and the range varies between 17 to 35 years. Almost similar observation has been made by Kulsum ${ }^{11}$, Zabin², Archibong et $\mathrm{al}^{4}$, Khan et $\mathrm{al}^{12}$. Another study by Parveen ${ }^{13} 65.0 \%$ of cases were between the ages of 26 to 35 years. All the above studies indicate that highest incidence of ectopic pregnancy in highly fertile period. The intrauterine pregnancy rate after radical surgery ranges between 50 and $70 \%$ with a 6 to $16 \%$ repeat ectopic pregnancy rate ${ }^{12}$. The prognosis for patients with an ectopic pregnancy is good for those with an early diagnosis. Thirty years ago when the diagnosis has been seldom made prior to rupture, the likelihood of a subsequent healthy term pregnancy is only approximately $30.0 \%$ cases. Currently the number is closer to $85.0 \%$ cases. The difference is that the earlier diagnosis and treatment, before the ectopicpregnancy can grow large enough to severely damage the tube ${ }^{13}$.

Among the risk factors identified in this present study, history of previous abortion or MR is $28.0 \%$ cases and history of pelvic infection $33.0 \%$ cases constitutes the major bulk. Patients with history of infertility are not also that much less and they are $25.0 \%$ cases and in this study only $3.0 \%$ patients have IUCD in situ when it is presented with ectopic pregnancy whereas Kulsuin ${ }^{19}$ has found $9.0 \%$ cases of IUCD users. Both Kulsum ${ }^{11}$ and $\mathrm{Zabin}^{2}$ have identified pelvic infection and past history abortion or MR and history of infertility as the main risk factor of ectopic pregnancy in this country. This situation reflects the status of low contraceptive practices in Bangladesh. In contrary the incidence of unsafe abortions by untrained indigenous abortion practitioner is quite high which increases the risk of ectopic pregnancy. The risk of ectopic pregnancy is 10 times higher in areas with a high incidence of illegal abortion and 6 times higher following clinical salpingitis ${ }^{14}$. Several case control studies have reported a strong association between ectopic pregnancy and Chlamydia trachomatis and Gonococcal infection ${ }^{12-15}$. Unfortunately, my patients were not screened for these organisms. In my study group, there was no patient having previous history of tubal surgery. However, in a current data analysis it has been shown that tubal surgery carries the highest risk of ectopic pregnancy ${ }^{13}$. In this study $6.0 \%$ patients are nulliparous and the peak incidence $(44.0 \%)$ is among the patients with para-2; then the incidence gradually declines with the increasing para. Kulsum ${ }^{11}$ has shown peak incidence of ectopic pregnancy among para-1 (37.0\% cases), para-2(25.0\% cases). Parveen ${ }^{13}$ and $\mathrm{Zabin}^{2}$ have also shown that the incidence is high among those who are para-2 or above. The ectopic pregnancy is closely related with high parity. In about $40.0 \%$ cases the etiology of ectopic pregnancy cannot be determined ${ }^{9}$. Thereafter, the most likely cause of ectopic pregnancy is delay in the passage of the fertilized ovum down the tube. Typically, the most important cause is damage to the ciliated epithelium and dysfunction in the tubal smooth muscle activity due to various reasons ${ }^{12}$.

The causes, risk factors and associated condition for ectopic pregnancy are evaluated.Pelvic inflammatory diseaseis widely recognized as the most important etiological factor?. Histopathological evidence of salphingitis is identified in $50.0 \%$ tubes harbouring a pregnancy ${ }^{11}$. Salpingitis causes peritubal adhesions, partial tubal lumen occlusion, intratubaladhesions, diverticula and disturbed tubal functions ${ }^{14}$. The salpingitis may be due to chlamydial, gonococcal, tubereculous, postabortal, puerperal or secondary to pelvic peritonitis; furthermore, infections source could be extragenital like appendicitis ${ }^{15}$. This is similar to the present study result.

In this present study, there are 3.0\% patients using IUCD, whereas in a study by Archibong et $\mathrm{al}^{4}$, it is $17.0 \%$ cases. Tubal pregnancy is more likely in women 
using IUCD. IUCD prevents intrauterine pregnancy hence the ratio of ectopic to intrauterine pregnancies is much higher ${ }^{7}$. Ectopic pregnancy is more likely with progesterone containing IUCDs rather than copper IUCDs. Copper has a cytotoxic and phagocytic effect on the sperm and oocytes. Use of progestin only pill or post-coital progestogen preparation increases the chance of tubal pregnancy probably by impaired tubal motility and polarity and does not always arrest ovulation hence lead to abnormal implantation ${ }^{11}$. Ectopic pregnancy is seen following sterilization surgery for blocked tubes and reversal of sterilization. The risk depends on the method and site of ligation, residual tubal length and adhesions and is also higher following cauterization procedures ${ }^{16}$. The fertilized ovum sometimes implants on the stump of a tube after partial salpingectomy.

After salpingoplasty and salpingostomy for distal tubal disease the incidence of ectopic pregnancy varies from 15 to $25.0 \%$ cases, probably because damage to the endosalpinx and the rate following reversal of tubal ligations is about $4.0 \%$ as the tubes have not been damaged by infection ${ }^{16}$. Assisted reproductive technique is also an important risk factors for ectopic pregnancy ${ }^{7}$. There is higher incidence of ectopic pregnancy after in vitrofertilization (IVF) and the risk seems to be between $2.0 \%$ to $5.0 \%$ higher $^{12}$. The first human pregnancy following IVF and embryo transfer was a tubal pregnancy. There is chance of combined gestation by the direct extrusion of embryos through the tubal ostium by hydrostatic forces.

\section{Conclusion}

In conclusion young age women are the most commonly suffering from ectopic pregnancy. However, it has been found that para 2 women are mostly suffering from ectopic pregnancy. Furthermore, pelvic infection or past history of abortion or MR are most commonly suffering from ectopic pregnancy.History of infertility isconstituted the main bulk of risk factors. Only few have history of tuberculosis and previous history of appendicitis, lower abdominal surgery. Previous use of IUCD are also vulnerable to ectopic pregnancy. Further large scale study should be conducted to get the real scenario.

\section{References}

1. Parashi S, Moukhah S, Ashrafi M. Main risk factors for ectopic pregnancy: a case-control study in a sample of Iranian women. International journal of fertility \& sterility. 2014;8(2):147-

2. Zabin F. Study on clinical presentations, management and operative findings of ectopic pregnancy. IPGMR Dhaka, 1998 [FCPS Dissertation].

3. Bouyer J, Coste J, Shojaei T, Pouly JL, Fernandez H, Gerbaud $\mathrm{L}$, et al. Risk factors for ectopic pregnancy: a comprehensive analysis based on a large case-control, population-based study in France. Am J Epidemiol. 2003;157(3):185-194

4. Archibong E, I Sobanda A. Ectopic pregnancy. In Abudhabi, Saudia Arabia. Saudia Medical Journal, 2000; Vol. 21(4): 330-334. 5. Selway J. The challenge of ectopic pregnancy. J Nurse Pract. 2006;2(9):583-591

6. Kriebs JM, Fahey JO. Ectopic pregnancy. J Midwifery Womens Health. 2006;51(6):431-439

7. Karaer A, Avsar FA, Batioglu S. Risk factors for ectopic pregnancy: A case-control study. Aust N Z J ObstetGynaecol. 2006;46(6):521-527

8. Coste J, Fernandez H, Joyé N, Benifla J, Girard S, Marpeau L, et al. Role of chromosome abnormalities in ectopic pregnancy. FertilSteril. 2000;74(6):1259-1260

9. Bouyer J, Rachou E, Germain E, Fernandez H, Coste J, Pouly $\mathrm{JL}$, et al. Risk factors for extrauterine pregnancy in women using an intrauterine device. FertilSteril. 2000;74(5):899-908

10. Barnhart KT, Sammel MD, Gracia CR, Chittams J, Hummel AC, Shaunik A. Risk factors for ectopic pregnancy in women with symptomatic first-trimester pregnancies. FertilSteril. 2006;86(1):36-43

11. Kulsum SU. Study on risk factors, clinical presentations and operative management of Ectopic pregnancy, IPGMR, Dhaka, 2002, [FCPS Dissertation]

12. Siddiqua S, Alam MM, Khan MAT. Ectopicpregnancy-A diagnostic dilemma, Bangladesh J ObstetGynaecol 2004; 19(1):7-10

13. Parveen R. Clinicopathological study of tubal ectopic pregnancy. A study of 60 cases, IPGMR, Dhaka 1992 [FCPS Dissertation]

14. Cornelius AC, Onyegbule A, Uchenna ET, Duke OA. A five year review of ectopic pregnancy at Federal Medical Centre, Owerri, South East, Nigeria. Nigerian journal of medicine: journal of the National Association of Resident Doctors of Nigeria. 2014;23(3):207-12.

15. Clayton HB, Schieve LA, Peterson HB, Jamieson DJ, Reynolds MA, Wright VC. Ectopic pregnancy risk with assisted reproductive technology procedures. Obstet Gynecol. 2006;107(3):595-604

16. Farquhar CM. Ectopic pregnancy. Lancet. 2005;366(9485):583-591 